\begin{tabular}{|c|c|c|c|}
\hline Eiszeitalter $u$. Gegenwart & 46 & $\begin{array}{c}91-98 \\
3 \mathrm{Abb} ., 1 \mathrm{Tab} .\end{array}$ & Hannover 1996 \\
\hline
\end{tabular}

\title{
Mengenbestimmung der jungen Abtragungsprodukte des Thüringer Waldes am Beispiel des Weser-Fluß-Systems
}

\author{
MarTin RaUSCH*)
}

Terrace, petrographic analysis, coarse gravel, gravel, crystalline rock, fluviatile erosion, tectonic uplift, porphyry,

\begin{abstract}
Kurzfassung: Es wird der Versuch unternommen, auf der Basis von Geröllanalysen die Menge der Abtragungsprodukte des Thüringer Waldes im Weser-Fluß-System zu bestimmen. Die errechnete Menge wird in Bezug gesetzt zu der Größe des Abtragungsgebietes. Daraus ergibt sich, daß innerhalb der letzten 200000 Jahre mindestens eine Schicht von $50 \mathrm{~m}$ Mächtigkeit abgetragen worden sein muß. Um die Höhe des Gebirges unverändert zu belassen, müßte eine junge Hebung von $1 / 4 \mathrm{~mm}$ pro Jahr angenommen werden. Solche Vorgaben sollten zu einer kritischen Diskussion Anlaß geben.
\end{abstract}

\section{[Amount of the young erosion-products of \\ the Thüringer Wald, located in the deposits of the Weser-river-system]}

Abstract: Analysis of pebble samples taken from terrace deposits of the Weser-River-System was applied to estimate the extent of young Thuringian Forest erosion. From the amount of Thuringian Forest material in the terrace deposits and the supposed dimension of the erosion area it can be calculated that within the last 200.000 years a hard rock layer of at least $50 \mathrm{~m}$ thickness was eroded. Assuming a constant altitude of the Thuringian Forest this means a tectonical uplift of approx. $1 / 4 \mathrm{~mm}$ per year. This results does not fit with the actual tectonical assumptions on the area and, therefore, should be discussed critically.

\section{Einleitung und Problemstellung}

Im Flußgebiet von Werra und Oberweser fallen die oftmals weite Flächen bildenden Kieskörper von Mittel- und Niederterrasse auf. Bemerkenswert ist der hohe Anteil von Geröllen aus Gesteinen des Thüringer Waldes. Ein Blick auf die geologische Karte zeigt: Ein relativ kleines Liefergebiet der Gerölle muß zu einem recht großen Sedimentationsraum in Beziehung gesetzt werden.

Die 1978 begonnenen Untersuchungen wurden im Jahre 1980 dankenswerter Weise durch eine Sachmittelbeihilfe der Deutschen Forschungsgemeinschaft unterstützt. Nach mehreren längeren Unterbrechungen konnten sie im Jahre 1995 wieder

\footnotetext{
*) Anschrift des Verfassers:

Dr. M. Rausch, Im Lohfeld 6, D-30989 Gehrden

E-mail: Dr.Rausch@T-Online.de

100520.3440@compuserve.com
}

aufgenommen und zu dem hier beschriebenen Abschluß gebracht werden. $\mathrm{Ob}$ sich alle in dieser Untersuchung angesprochenen Fragen in Zukunft werden beantworten lassen, bleibt abzuwarten. Trotzdem wird erstmals der Versuch gemacht, die zu Beginn beschriebene qualitative Feststellung zu quantifizieren. Dabei können natürlich numerische Aussagen nur so gut sein wie das zu Grunde gelegte Datenmaterial. Für den Bereich der ehemaligen DDR mußte auf Literaturangaben sowie auf das Kartenwerk aus dem Jahre 1945 zurückgegriffen werden. Kurze Studienreisen in den vormals nicht zugänglichen Raum der neuen Bundesländer sowie die stichprobenhafte Überprüfung der Geröllführung der fraglichen Sedimente zeigen eine sehr gute Übereinstimmung mit den Angaben aus der Literatur. Daher kann von einer weiteren Beprobung abgesehen werden.

Das gefundene Ergebnis erscheint in seiner Größenordnung so überraschend, daß eine kontroverse Diskussion unumgänglich sein dürfte.

\section{Berechnung der Abtragungsmenge des Thüringer Waldes}

Um die fragliche Abtragungsmenge für die Zeit vom Beginn der Mittelterrassensedimentation bis heute zu berechnen, sind zwei Vorbedingungen zu definieren:

1. Das Abtragungsgebiet (siehe Abb. 1) darf in dieser Zeit seine Ausdehnung nicht wesentlich verändert haben.

Diese Bedingung ist erfüllt. Der „Ausbiß von Gesteinen des Rotliegenden und älterer Formationen" kann sich zwangsläufig nicht entscheidend verändert haben. Die "heutige Wasserscheide“, die das Abtragungsgebiet nach NE begrenzt, soll ebenfalls für diese Zeit des Quartärs seine Lage nur unwesentlich verschoben haben (EIssmanN 1975). Allerdings scheint die Frage der Korrelation der Mittelterrasse der Weser mit den Mittelterrassen in unmittel- 


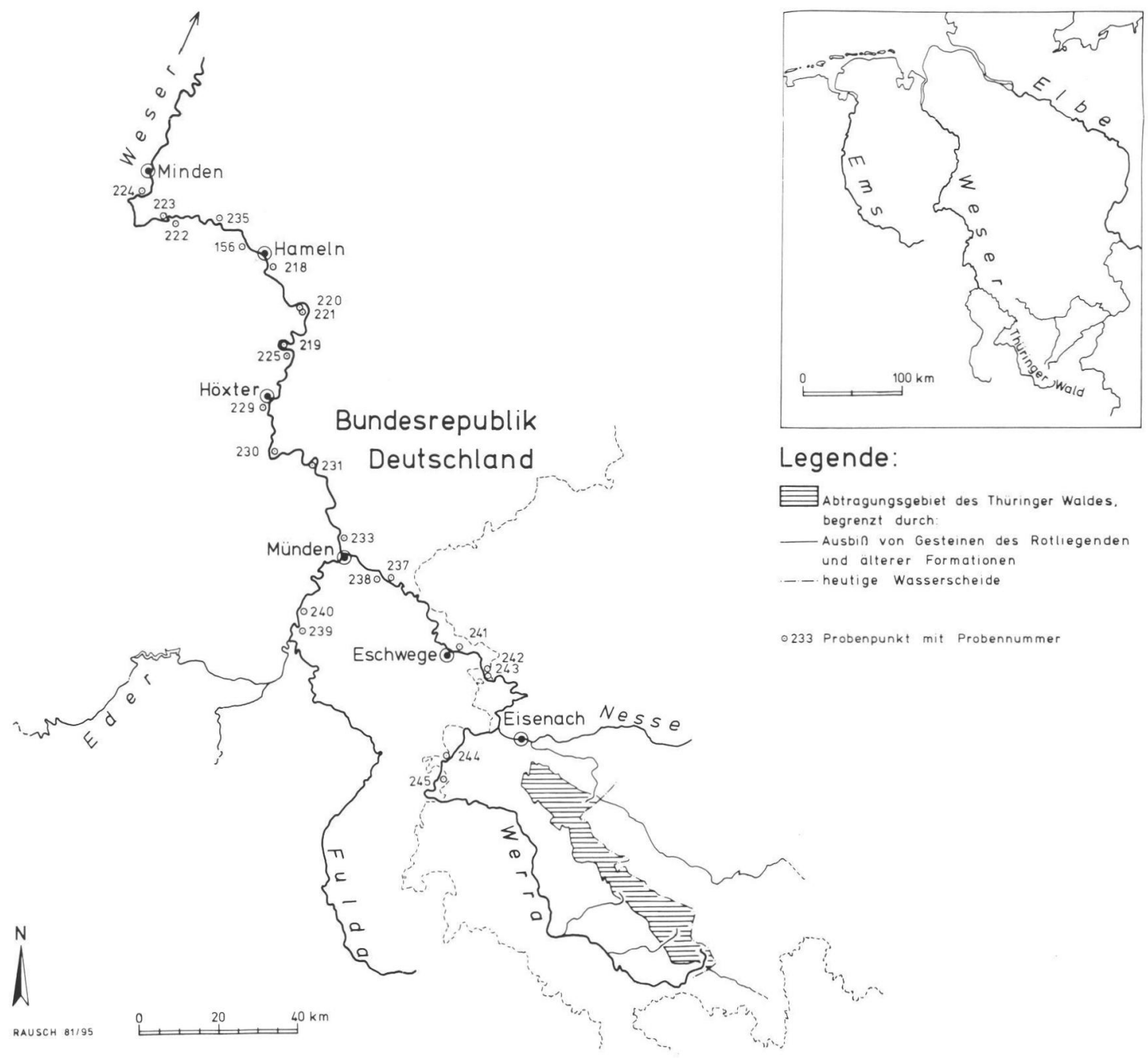

Abb. 1: Übersichtskarte, Abtragungsgebiet des Thüringer Waldes, Lage der Kiesproben

Fig. 1: Sketch map, erosion area of the Thüringer Wald, location of gravel samples

barer Nachbarschaft des Thüringer Waldes noch teilweise offen. Für die dortige ,Jüngere Mittelterrasse" ist ein Drenthezeitliches Alter, wie für ihr Äquivalent an der Weser, anzunehmen. Für diesen Zeitabschnitt kann auch mit einem gleich großen Einzugs- und damit Abtragungsgebiet der Flüsse Hörsel und Nesse gerechnet werden. (Freundliche mündliche Mitteilung von H. SCHramm vom 4. 12. 1995.) In den älteren Abschnitten des Quartärs war das Einzugsgebiet größer (SCHRAMm 1965).

2. Größere Umlagerungen aus älteren Oberterrassenkiesen in die jüngeren Mittel- und Niederterrassenablagerungen dürfen nicht stattgefunden haben.
Auch diese Bedingung ist erfüllt. Vor Ablagerung der Mittelterrasse müssen die Täler bis fast auf ihre heutige Festgesteinssohle ausgeräumt worden sein. Dieser Erosion fielen wohl auch die älteren Flußablagerungen zum Opfer. So erklärt sich ihr heutiger bescheidener Flächenanteil im Einzugsgebiet von unter $5 \%$. Da es sich hierbei zu einem erheblichen Teil nur um dünne Restschotterdecken handelt, muß ihr Raumanteil sicherlich mit weit unter $1 \%$ angenommen werden. Niemals ist in der Literatur des Berglandes (außerhalb von Subrosionsgebieten) von Oberterrassenvorkommen im Liegenden der Mittelterrasse berichtet worden. Der einzige dokumentierte Befund, bei dem die Mittelterrasse über älterem Quartär (Holstein-Interglazial) liegt, ist die 
Zeche Nachtigall bei Holzminden (zuletzt KLOSTERMANN in BENDA 1995). Aber auch dieses Vorkommen muß in einem Subrosionszusammenhang gesehen werden.

Dem widersprechen auch nicht die Ausführungen RoHDE's (1989). Die sehr selten höheren Mächtigkeiten älterer Terrassenablagerungen im Bereich der Oberweser sind zumeist auf tektonische Sonderfälle beschränkt (Preuss 1975).

Für die jüngeren Terrassenablagerungen liegen die Verhältnisse grundlegend anders. In der Saale-Kaltzeit wurde der Mittelterrassenkörper aufgeschüttet. Innerhalb des Vereisungsgebietes wird er überlagert von der Grundmoräne des Drenthe-Stadiums der Saale-Vereisung. Der Terrassenkörper, dessen Oberfläche ca. 20 m über der heutigen Talaue liegt, läßt sich auf Grund seiner oft markanten Ausbildung gut in das vormals nicht vereiste Gebiet hinein verfolgen. Regionale Probleme mit seiner stratigraphischen Ansprache (Rohde 1989) widersprechen dem nicht.

Die folgende Erosionsphase räumte aber nur geringe Teile dieser Körper wieder aus. So standen sie bei der folgenden Akkumulation der Niederterrasse in großem Umfang als Materiallieferant zur Verfügung. Entsprechende Umlagerungen konnten im Flußgebiet der Leine auch geröllanalytisch nachgewiesen werden (Rausch 1978).

Von diesen Überlegungen ausgehend wurden im Laufe der Jahre etliche Proben im Untersuchungsgebiet entnommen (vgl. Abb. 1). Deren Mittelkiesfraktion wurde geröllanalytisch ausgewertet, wobei jeweils ca. 800 Gerölle gezählt wurden (zur genauen Methodik siehe Rausch 1977). Die Zusammensetzung der Mittelkiesfraktion wird dabei als repräsentativ für die Zusammensetzung der Gesamtprobe angesehen.

Aus den so gewonnenen Werten wurde, zusammen mit den aus der Literatur bekanntgewordenen Zählungen, eine Kurve konstruiert, die den Gehalt an Geröllen aus Gesteinen des Thüringer Waldes, bezogen auf den Flußkilometer des Werra-WeserSystems, darstellt (siehe Abb. 2).

Einige Zählungen von Fremdautoren mußten modifiziert werden. So konnten von den Analysen WORTMANN's (1968: 231) nur Mittelwerte seiner „Südlichen Petrofazies" berücksichtigt werden. Bei den Angaben Plischke's (1976) wurden die glazigenen Einflüsse numerisch beseitigt.

Abb. 2: Gehalt an Geröllen des Thüringer Waldes, bezogen auf den Flußkilometer des Werra-WeserSystems

Fig. 2: Concentration of pebbles of the Thüringer Wald as a function of the river-kilometers of the WerraSystem 
Zuerst soll hier der Flußbereich bis zur Porta Westfalica betrachtet werden, da für diesen Abschnitt repräsentative Proben vorliegen. Die Kurve zeigt den vermuteten Zusammenhang: Mit zunehmender Entfernung vom Liefergebiet nimmt der Anteil von Geröllen aus Gesteinen des Thüringer Waldes ab.

Dieses Phänomen wird von der erneuten Zunahme von Thüringer-Wald-Geröllen auf dem Flußabschnitt zwischen Hameln (Flußkilometer 136) und Minden (Flußkilometer 210) überlagert. Die Erklärung fällt um so leichter, als in diesem Bereich des Tales niemals Oberterrassenkiese der Weser abgelagert wurden, die dann später hätten umlagert werden können. Vielmehr muß der Fluß in der fraglichen Zeit noch von Hameln aus über Coppenbrügge in die Leine geflossen sein (LÜTTIG 1960; RAUSCH 1977: 51; RHODE 1983).

Kurz vor Hameln tritt bekanntlich die Weser aus einem überwiegend engen in ein nun auf lange Strecken sehr weites Tal ein. Damit stehen dem Fluß auch nicht mehr derartig große Mengen anstehendes Festgestein zur Abtragung und anschließendem Einbau in seine Kieskörper zur Verfügung. Zwangsläufig kommt es beim weiteren Transport zu einer Anreicherung der härteren Bestandteile aus Geröllen des Paläozoikums. Die gleiche Gesetzmäßigkeit wurde bereits für die Sedimente von Leine und Innerste beschrieben (RAusch 1997: 36).

Der nächste Arbeitsschritt diente der planimetrischen Bestimmung der Flächenausdehnung der untersuchten Terrassenkörper. Als Grundlage dafür wurden, soweit möglich, orohydrographische Karten im Maßstab 1:50 000, sonst normale topographische Karten 1:25000 herangezogen.

Für die Niederterrasse wurde die Fläche der heutigen Talniederung bestimmt. Für die Mittelterrasse wurde angenommen, daß sie die rezenten Täler nach Abschluß ihrer Sedimentation bis zu einer Höhe von ca. $20 \mathrm{~m}$ über der heutigen Talaue ausfüllte. Diese gedachte Fläche wurde ebenfalls planimetriert. Es sei hier ausdrücklich angemerkt, daß besonders bei der Bestimmung der Mittelterrassenfläche äußerst zurückhaltend vorgegangen wurde. Nebentaleinschnitte blieben unberücksichtigt. Im sehr engen Oberlauf der Flüsse wurde an einigen Stellen für Mittel- und Niederterrasse der gleiche Flächeninhalt angenommen. Das Ergebnis dieser Bemühungen ist in Tabelle 1, Spalten 1 und 3, festgehalten.

Auf diesen Werten aufbauend, wurden nach Literaturdaten sowie der Durchsicht diverser Bohrprotokolle durchschnittliche Mächtigkeiten für die beiden Terrassenkörper festgelegt, um ihren Rauminhalt zu bestimmen. Für die Niederterrasse wurde der Flächeninhalt mit $10 \mathrm{~m}$ mulitpliziert (Tabelle 1, Spalte 2), für die Mittelterrasse mit $20 \mathrm{~m}$ (Spalte 4). Für letztere wird aus dem Oberlauf der Weser aber auch über höhere Mächtigkeiten berichtet. Auf Blatt Uslar beträgt die Gesamtmächtigkeit des Schotterkörpers ca. 30 m (LEPPER 1977).

Trotzdem sind diese Werte in den unmittelbaren Randbereichen der Terrassenkörper etwas zu hoch gegriffen. Andererseits muß an anderer Stelle die Mächtigkeit duch die zahlreichen Subrosionssenken im Untergrund erheblich höher sein. Auf dem Gebiet Thüringens nimmt ihr Flächenanteil einen sehr großen Raum ein (Ellenberg 1969, 1972; Hoppe 1974). Aber auch für den Bereich der alten Bundesländer wurde darüber berichtet (u. a. LEPPER 1976). Die dabei eingelagerten Sedimentfüllungen können $100 \mathrm{~m}$ Schichtdicke überschreiten. Eine quantitative Behandlung dieser Senken ist wegen des nur fragmentarisch vorliegenden Untersuchungsmaterials nicht möglich. Es wird daher postuliert, daß dieses Material die teilweise geringeren Mächtigkeiten der Kieskörper an ihren Rändern gerade ausgleicht. Obwohl diese Annahme die Gesamtabtragungsmenge nach Meinung des Autos sicher negativ beeinflußt, ist kein anderer numerischer Ansatz möglich.

Eine Umlagerung von Mittelterrassenkies in der Niederterrasse wird als wahrscheinlich angesehen. Der geröllanalytische Beweis konnte, bedingt durch die spezielle Materialzusammensetzung der Proben, für die Weser hier nicht geführt werden. Er gelang aber an anderer Stelle für die Ablagerungen der Leine (Rausch 1978). Aus diesem Grunde werden die Ablagerungen von Mittel- und Niederterrasse hier zusammen betrachtet (Tab. 1, Spalte 5), die Rauminhalte wurden addiert.

Nun wurde mit Hilfe der Verteilungskurve aus Abb. 2 für den Bereich jeder topographischen Karte ein Mittelwert für den Anteil an Thüringer-WaldMaterial festgelegt (Tab. 1, Spalte 6). Durch Verrechnung der Spalten 5 und 6 ergibt sich die Menge an Thüringer-Wald-Material pro topographische Karte in $\mathrm{km}^{3}$ (Spalte 7).

Werden die einzelnen Zahlenwerte addiert, so erhält man für den Bereich zwischen dem Austritt der einzelnen Lieferflüsse aus dem anstehenden ThüringerWald-Kristallin und der Straßenbrücke an der Porta Westfalica bei Minden einen Betrag von $8,2 \mathrm{~km}^{3}$ abgetragenen Thüringer-Wald-Materials.

Im Flachland nördlich der Porta Westfalica ist man auf Hochrechnungen angewiesen, da die glazigene Beeinflussung des Raumes das Bild verschleiert. Auch in neuerer Zeit bekannt gewordene Zählungen (u. a. PlischKe 1976, Fricke 1988) können nur bedingt zu Hilfe genommen werden. Es erscheint auf der anderer Seite unwahrscheinlich, daß die Sedimentation von Thüringer-Wald-Material mehr oder weniger plötzlich mit Austritt der Weser durch die Porta Westfalica ins Flachland endete. So wurde versucht, die in Abb. 2 dargestellte Kurve nach Norden hin zu verlängern bis zu einem Punkt, an dem dieser 
Tab. 1: Rauminhalt der Flußterrassen von Werra und Weser Table 1: Volume of the terraces of the Rivers Werra und Weser

Topographische

Karten nach Flüssen geordnet

Weser

L3718 Minden

3819 Vlotho

L3970 Rinteln

L3720 Stadthagen

L3922 Hameln

L4122 Holzminden

L4322 Höxter

L4522 Münden

Summe

\section{Werra}

L4524 Göttingen

L4724 Witzenhausen

L4726 Heiligenstadt

L4926 Eschwege

4928 Mihla

L5126 Eisenach West

L5128 Bad Hersfeld

L5326 Tann

5228 Schmalkalden

5328 Wasungen

L5528 Meiningen

5530 Hildburghausen

5531 Eisfeld

Summe

Nebenflüsse Werra

ohne Hörsel-System

5228 Schmalkalden

5329 Zella-Mehlis

5330 Suhl

5430 Schleusingen

L5528 Meiningen

5530 Hildburghausen

Summe

Nebenflüsse Hörsel-S.

L5126 Eisenach West

5028 Eisenach Ost

5029 Fröttstedt

5128 Rhula

5129 Waltershausen

Summe

\begin{tabular}{|c|c|c|c|c|c|c|}
\hline 1 & SP 2 & SP 3 & SP 4 & & SP6 & SP 7 \\
\hline & $T / \mathrm{km}^{3}$ & & $\mathrm{MT} / \mathrm{km}^{3}$ & & $\begin{array}{l}\text { Stück \% } \\
\text { TW }\end{array}$ & \\
\hline
\end{tabular}

$\begin{array}{lllllll}16,875 & 0,16875 & 56,45 & 1,129 & 1,29775 & 22 & 0,2855 \\ 16,363 & 0,16363 & 21,394 & 0,42788 & 0,59151 & 21 & 0,1242 \\ 72,8 & 0,728 & 88,675 & 1,7735 & 2,5015 & 18 & 0,4503 \\ 7,325 & 0,07325 & 8,775 & 0,1755 & 0,24875 & 20 & 0,0498 \\ 35,475 & 0,35475 & 54,6 & 1,092 & 1,44675 & 16 & 0,2315 \\ 37,95 & 0,3795 & 57,925 & 1,1585 & 1,538 & 16 & 0,2461 \\ 49,2 & 0,492 & 65,6 & 1,312 & 1,804 & 19,5 & 0,3518 \\ 19,275 & 0,19275 & 27,525 & 0,5505 & 0,74325 & 24,5 & 0,1821 \\ & & & & & & 1,9213\end{array}$

$\begin{array}{lll}1,825 & 0,01825 & 2,25 \\ 27,725 & 0,27725 & 66,2 \\ 11,925 & 0,11925 & 12,525 \\ 54,1 & 0,541 & 68,65 \\ 0,3063 & 0,00306 & 0,7563 \\ 48,6 & 0,486 & 70,975 \\ 6,125 & 0,06125 & 8 \\ 8,75 & 0,0875 & 11,2 \\ 7,4313 & 0,07431 & 9,6563 \\ 7,5625 & 0,07563 & 8,3812 \\ 17,575 & 0,17575 & 21,725 \\ 5,2188 & 0,05219 & 9,9188 \\ 3,8125 & 0,03813 & 7,9875\end{array}$

0,045
1,324
0,2505
1,373
0,01513
1,4195
0,16
0,224
0,19312
0,16763
0,4345
0,19838
0,15975

0,06325

1,60125

0,36975

1,914

0,01819

1,9055

0,22125

0,3115

0,26744

0,24325

0,61025

0,25056

0,19788
0,0171

0,5124

0,1368

0,9092

0,0086

1,3053

0,1438

0,2461

0,218

0,208

0,5675

0,233

0,1979

4,7037

$\begin{array}{lllllll}6,3938 & 0,06394 & 6,4875 & 0,12975 & 0,19369 & 90 & 0,1743 \\ 6,025 & 0,06025 & 6,025 & 0,1205 & 0,18075 & 90 & 0,1627 \\ 0,45 & 0,0045 & 0,45 & 0,009 & 0,0135 & 90 & 0,0123 \\ 4,5438 & 0,04544 & 4,8813 & 0,09763 & 0,14306 & 90 & 0,1288 \\ 4,8 & 0,048 & 4,8 & 0,096 & 0,144 & 90 & 0,1296 \\ 5,1688 & 0,05169 & 7,1813 & 0,14363 & 0,19531 & 90 & 0,1758 \\ & & & & & & 0,7835\end{array}$

$\begin{array}{lllllll}\mathbf{5 , 3 7 5} & 0,05375 & 7,25 & 0,145 & 0,19875 & 50 & 0,0994 \\ 7,8813 & 0,07881 & 11,375 & 0,2275 & 0,30631 & 60 & 0,1838 \\ 7,1938 & 0,07194 & 21,425 & 0,4285 & 0,50044 & 75 & 0,3753 \\ 0,6875 & 0,00688 & 0,6875 & 0,00138 & 0,02063 & 90 & 0,0186 \\ 4,25 & 0,0425 & 4,25 & 0,085 & 0,1275 & 90 & 0,1148 \\ & & & & & & 0,7919\end{array}$

Endsumme:

8,2004

theoretische Fluß kein Thüringer-Wald-Material in der Mittelkiesfraktion mehr führt. Drei denkbare Möglichkeiten (Abb. 2, Kurven 1, 2, 3) wurden in
Anlehnung an den sicher nachgewiesenen Kurvenverlauf zwischen Flußkilometern 0 und 250 durch Extrapolation ermittelt. 
Konsequenter Weise wurden nun für diesen hypothetischen Fluß die durchschnittlichen Terrassenrauminhalte des untersuchten Berglandes berechnet. Die Terrassenbreite wurde mit $25 \mathrm{~km}$, die Mächtigkeit der Niederterrasse mit $10 \mathrm{~m}$ und die Mächtigkeit der Mittelterrasse mit $20 \mathrm{~m}$ angenommen. So ergeben sich für

$$
\begin{aligned}
& \text { Kurve 1: } 3,4 \mathrm{~km}^{3} \\
& \text { Kurve 2: } 4,6 \mathrm{~km}^{3} \\
& \text { Kurve 3: } 5,9 \mathrm{~km}^{3}
\end{aligned}
$$

Abtragungsprodukte des Thüringer Waldes, bezogen auf die Mittelkiesfraktion.

Das zur Verfügung stehende Abtragungsgebiet (Abb. 1) hat eine planimetrisch bestimmte Flächenausdehnung von ca. $512 \mathrm{~km}^{2}$. Setzt man das Ablagerungsgebiet dazu in Beziehung, indem man die abgetragenen Mengen in Form einer ebenen Schicht auf das anstehende Festgestein stapelt, so ergibt sich die folgende Schichtdicke: Bei Berücksichtigung lediglich der unmittelbar zugänglichen Sedimente zwischen dem Rand des Thüringer Waldes und der Porta Westfalica bei Minden $=16,02 \mathrm{~m}$.

Für die drei Kurven aus Abb. 2 jeweils ca.

$$
\begin{aligned}
& \text { Kurve } 1=6,63 \mathrm{~m} \\
& \text { Kurve } 2=8,96 \mathrm{~m} \\
& \text { Kurve } 3=11,51 \mathrm{~m} .
\end{aligned}
$$

Im Folgenden soll von einer mittleren Mächtigkeit, also von Kurve 2 ausgegangen werden. Zusammen ergibt das eine Schichtdicke von 24,98 m. Dieser Betrag ist sicherlich noch zu klein, wenn die nicht mehr im Untersuchungsgebiet vorhandenen, sondern ins Flachland transportierten feinklastischen Flußsedimente in die Überlegungen einbezogen werden. Dieses ließe sich leicht durch den Vergleich von Korngrößenanalysen aus den Flußterrassen mit denen von Hangschuttmassen über anstehendem Porphyr und solchen aus temporären „Klärbecken“, z. B. den aufgeschlossenen Subrosionsbildungen der „Zeche Nachtigall", erreichen.

Das zur Verfügung stehende Archivmaterial wurde ausgewertet und in Abb. 3 zusammengefaßt. Die aufgeworfene Fragestellung ist auf diese Weise natürlich nicht abschließend zu klären. Immerhin trifft die in Abb. 3, Kurve A, dargestellte Zusammensetzung sehr schön das Verteilungsbild, das bei allen derartigen kaltzeitlichen Flußablagerungen zu erwarten ist. Eine denkbare Korngrößenabnahme der Ablagerungen in Transportrichtung zwischen Münden und Minden konnte nicht beobachtet werden. Die fraglichen Terrassenkiese sehen überall recht ähnlich aus. Größere Einschaltungen feinkörniger Bestandteile sind bei normaler Lagerung nicht vorhanden.

Auf der anderen Seite wird aus Analogieschlüssen zu den Harzporphyren angenommen, daß - geschätzt - schon bei ihrer Verwitterung im Anstehenden bereits weit über die Hälfte des Materials als
Schluff anfällt. Dieses Material muß zu seinem überwiegenden Teil erst außerhalb des untersuchten Sedimentationsgebietes zur Ablagerung gekommen sein.

Wenn diese Ausführungen richtig sind, ist es sicherlich angemessen, die Dicke der abgetragenen Schicht auf $50 \mathrm{~m}$ zu verdoppeln. Diese Schätzung wird vom Autor noch als ausgesprochen vorsichtig angesehen. Ebenso bleibt die sicher vorhanden gewesene chemische Lösung der Gesteine unberücksichtigt.

\section{Diskussion des Ergebnisses und der möglichen tektonischen Konsequenzen}

Bereits während des gesamten Tertiärs, natürlich ebenso in der Zeit des Quartärs, war der Thüringer Wald Festland und damit Abtragungsgebiet. Wenn wir heute immer noch ein stattliches Gebirge mit durchschnittlichen Höhen zwischen $800 \mathrm{~m}$ und $900 \mathrm{~m}$ ü. NN vor uns haben, so beweist das, daß die „epirogenen Hebungen“" größer waren als der Abtragungsbetrag. Für die Zeit vom Beginn der Mittelterrassensedimentation bis heute wurde eine abgetragene Schicht von $50 \mathrm{~m}$ Mächtigkeit postuliert.

Diese Abtragung ging sicherlich nicht so schematisch flächenhaft vor sich, dennoch soll hier die Frage aufgeworfen werden, ob einem entsprechenden Erosionsbetrag nicht ein zumindest ebenso großer junger Hebungsbetrag entgegengestellt werden muß. Der Thüringer Wald ist ja auch heute noch als mächtiges Gebirge erhalten!

Im folgenden wird die postulierte Dicke der abgetragenen Schicht in Beziehung zu der zur Verfügung stehenden Zeit gesetzt.

Das Ergebnis wäre eine junge Hebung von $0,25 \mathrm{~mm} / \mathrm{Jahr}$ für die letzten 200000 Jahre, vorausgesetzt, man will nicht ein noch geringeres Mittelterrassenalter annehmen.

Eine derartig große Hebung von 1/4 mm pro Jahr für ein Gebiet wie den Thüringer Wald, also außerhalb eines jungen Faltengebirges, erscheint auf den ersten Blick sehr ungewöhnlich! Sie wird allerdings durch geodätische Messungen untermauert. So beschreibt ElLENBERG (1993) rezente vertikale Erdkrustenbewegungen aus Thüringen, die für den $\mathrm{Ab}$ tragungsraum Beträge von bis zu 2,0 mm/Jahr erreichen. Der Beobachtungszeitraum, der seiner Untersuchung zu Grunde liegt, beträgt ca. 20 Jahre. Der Thüringer Wald tritt dabei auch rezent als Horst in Erscheinung.

Darüber hinaus bemerkt EllENBERG (freundliche Mitteilung vom 10. 11. 1995): „Man muß mit einer aktiven Störungstektonik an den Rändern des Thüringer Waldes während des gesamten Quartärs rechnen, die morphogenetisch relevanten Beträge sind jedoch mit Sicherheit während des Tertiärs erzeugt worden." 


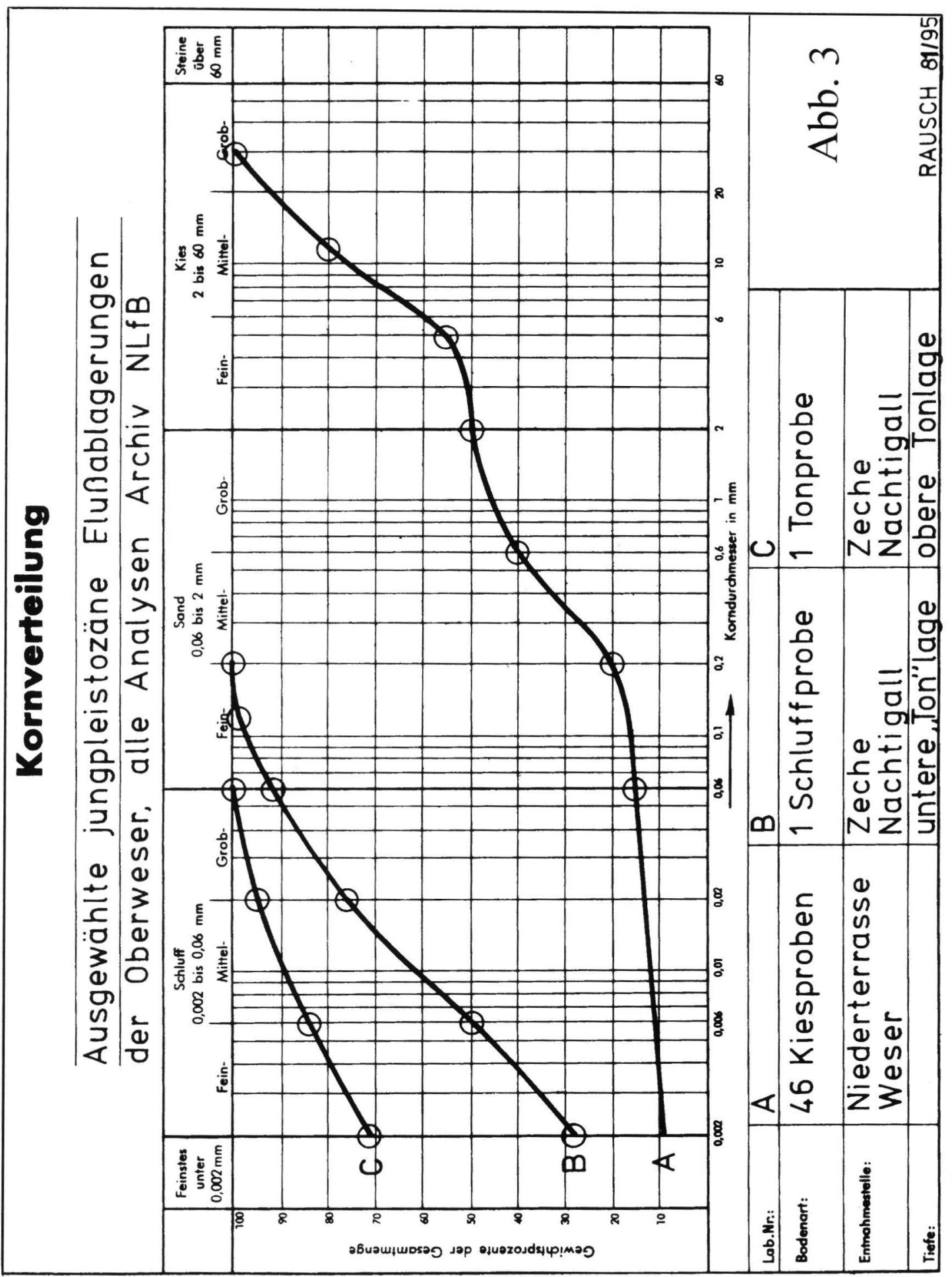


Trotzdem soll hier einmal der ermittelte Jahreshebungsbetrag auf die gesamte Quartärzeit ausgedehnt werden. Bei einem angenommenen Alter des Quartärs von 2,3 Millionen Jahren ergibt sich ein Hebungsbetrag von $575 \mathrm{~m}$. Dieser Wert liegt noch unterhalb der heutigen Höhenlage des Gebirges, hat aber die gleiche Größenordnung.

Untermauert wird dieses Ergebnis durch das Fehlen von Thüringer-Wald-Geröllen in den Schichten des norddeutschen Pliozäns, die älter sind als Reuver (freundliche mündliche Mitteilung von BARTHOLOMÄUs, 10/95). Die junge Hebung des Thüringer Waldes und die damit verbundene Abtragung müssen also entweder frühestens hier eingesetzt haben, oder entsprechende Gerölle sind der Verwitterung zum Opfer gefallen oder noch nicht aufgefunden worden.

\section{Schriftenverzeichnis}

EISSMANN, L. (1975): Das Quartär der Leipziger Tiefland-Bucht und angrenzender Gebiete um Saale und Elbe. - Schriftenr. geol. Wiss., 2, 58 Abb., 23 Tab., 17 Taf., 1-263; Berlin.

ELLENBERG, J. (1969): Die geologisch-morphologische Entwicklung des südwest-thüringischen Werragebietes im Pliozän und Quartär. - Unveröff. Diss., Univ. Jena; Jena.

- (1972): Auslaugung und Sedimentation in Gebiet von Gerstungen. - Geol., 21, Nr. 3, S. 296-304, 3 Abb.; Berlin.

- (1993): Rezente vertikale Erdkrustenbewegungen in Thüringen. - Jenaer Geogr. Schriften, 1, 7-22; Jena.

FRICKE, K. (1988): Zur Hydrogeologie der Stadt Lübbecke. - Unveröff. Diplomarbeit Geol. Inst. Univ. Hannover; Hannover.

Hoppe, W. (1974): Geologie von Thüringen; Gotha/Leipig.
Klostermann, J. (1995): IV. Nordrhein-Westfalen. In: Benda, L. (Hrsg.): Das Quartär Deutschlands. - Borntraeger; Stuttgart.

LEPPER, J. (1976): Erläuterungen zu Blatt 4322 Karlshafen. - Geol. Kt. Nordrh.-Westf., 190 S., 14 Abb., 11 Tab., 6 Taf.; Krefeld.

- (1977): Erläuterungen zu Blatt Uslar Nr. 4323. - Geol. Kt. Niedersachsen, 129 S., 14 Abb., 13 Tab., 10 Taf., Hannover.

LüTTIG, G. (1960): Neue Ergebnisse quartärgeologischer Forschung im Raum Alfeld-Hameln-Elze. Geol. Jb., 77, 337-390; Hannover.

PLISCHKE, I. (1976): Untersuchungen zur Geröll- und SchwermineralFührung fluviatiler und glazifluviatiler Pleistozänkiese im Raum Nienburg/Weser.- Unveröff. Diplomarbeit Geol. Inst. Univ. Hannover, 43. S., ca. 300 Tab., 6 Anl.; Hannover.

Preuss, H. (1975): Gliederung und Zusammensetzung der Weserterrassen-Körper bei Bodenfelde (mit einer geologischen Kartierung). - Mitt. Geol. Inst. Techn. Univ. Hannover, 12, 1-49, Hannover.

Rausch, M. (1977): Fluß-, Schmelzwasser- und Solifluktionsablagerungen im Terrassengebiet der Leine und Innerste - ein Beitrag zur pleistozänen Flußgeschichte Südniedersachsens. - Mitt. Geol. Inst. Techn. Univ. Hannover, 14, 1-84; Hannover.

- (1978): Umlagerungsvorgänge bei der Sedimentation eiszeitlicher Flußablagerungen - ein Beispiel aus dem Terrassengebiet der Leine SE Hannover. Z. dt. geol. Ges., 129, 1-5, 3 Abb., 1 Tab.; Hannover.

RHodE, P. (1989): Elf pleistozäne Sand-Kies-Terrassen der Weser: Erläuterungen eines Gliederungsschemas für das obere Wesertal. - Eiszeitalter u. Gegenwart, 39, 42-56; Hannover.

- (1983): Quartär. - Erl. geol. Kt. Niedersachsen 1: 25000, Bl. 3724 Pattensen: 42-109, Abb. 12-39, Tab. 4-16; Hannover.

Sснгамм, H. (1965): Untersuchungsergebnisse zur altpleistozänen Flußgeschichte im nördlichen Vorland des Thüringer Waldes. - Wiss. Zeits. der Friedrich-Schiller-Univ., Mathem.-Naturwiss. Reihe, 4, Jg. 14; Jena.

Wortmann, H. (1968): Die morphogenetische Gliederung der Quartärbasis des Wiehengebirgsvorlandes in Nordwestdeutschland. - Eiszeitalter u. Gegenw., 19, 227-239; Öhringen.

Manuskript eingegangen am 15. 11. 1994 